EESTI NSV TEADUSTE AKADEEMIA TOIMETISED, 26. KÖIDE

KEEMIA * GEOLOOGIA. 1977, Nr. 2

НЗВЕСТИЯ АКАДЕМИИ НАУК ЭСТОНСКОЙ ССР. ТОМ 26

ХИМНЯ * ГЕОЛОГИЯ. 1977 , № 2

\title{
РАВНОВЕСНАЯ РАСТВОРИМОСТЬ ХЛОРИСТОГО ВОДОРОДА В РЕАГИРУЮЩИХ С НИМ СИСТЕМАХ ИЗ ПИПЕРИЛЕНА И ЕГО ГИДРОХЛОРИДОВ
}

Гидрохлорирование жидких алкадиенов, в том числе пиперилена, сухим хлористым водородом находит применение в разных технологических процессах производства высокомолекулярных соединений, душистых и поверхностноактивных веществ. При технологических расчетах по проектированию реакторов для гидрохлорирования необходимо знать связь между равновесными концентрациями хлористого водорода в жидкой и газовой фазах. Такая зависимость в упрощенном виде обычно выражается законом Генри

$$
p_{i}^{p}=E \cdot x_{i}^{p},
$$

где $p_{i}^{p}-$ равновесное парциальное давление абсорбируемого компонента в газовой фазе, находящегося в контакте с жидкостью, атм; $x_{i}^{p}-$ содержание того же компонента на поверхности жидкости, моль/моль; $E-$ константа Генри, атм.

К этому закону следует добавить уравнения, выражающие зависимость константы Генри от температуры и концентрации других компонентов, кроме хлористого водорода в жидкой фазе.

Численное значение константы Генри опредєляется обычно по экспериментальным данным путем измерения концентраций компонентов в обеих равновесных фазах, поскольку в случае физической абсорбции при достижении равновесного состояния концентрации компонентов в глубине фазы и на поверхности ее одинаковы. При абсорбции, сопровождаемой химической реакцией в жидкости и испарением компонентов жидкости, как это имеет место в данном случае, непосредственное экспериментальное определение равновесных концентраций невозможно, так как полное равновесие при этом не достигается.

Определение растворимости газов в жидкостях, с которыми они реагируют, успешно проводится на аппарате с ламинарной струей $\left[{ }^{1,2}\right]$, но из-за очень кратковременного контакта между жидкостью и газом этот аппарат более пригоден для исследования быстрых реакций и не применим в случае медленных реакций.

Сложный, а иногда и неопределенный режим течения жндкостной пленки затрудняет анализ экспериментальных данных на аппаратах пленочного типа. Главным недостатком аппарата барботажного типа является отсутствие точного определения поверхности контакта между фазами. Несмотря на это, барботажный аппарат кажется достаточно подходящим для исследования абсорбции с одновременной медленной 
реакцией, поскольку в таком случае можно провести опыт так, что измеряемая концентрация в объеме жидкости сравнительно мало отличается от равновесной.

\section{Методика опытов}

Опыты по изучению растворимости хлористого водорода в пиперилене, его гидрохлоридах и различного состава их смесях проводились в лабораторном стеклянном реакторе периодического действия барботажного типа при стационарном изотермическом режиме. Сухой газообразный хлористый водород получали из соляной кислоты под действием концентрированной серной кислоты. Схема опытной установки приведена на рисунке.
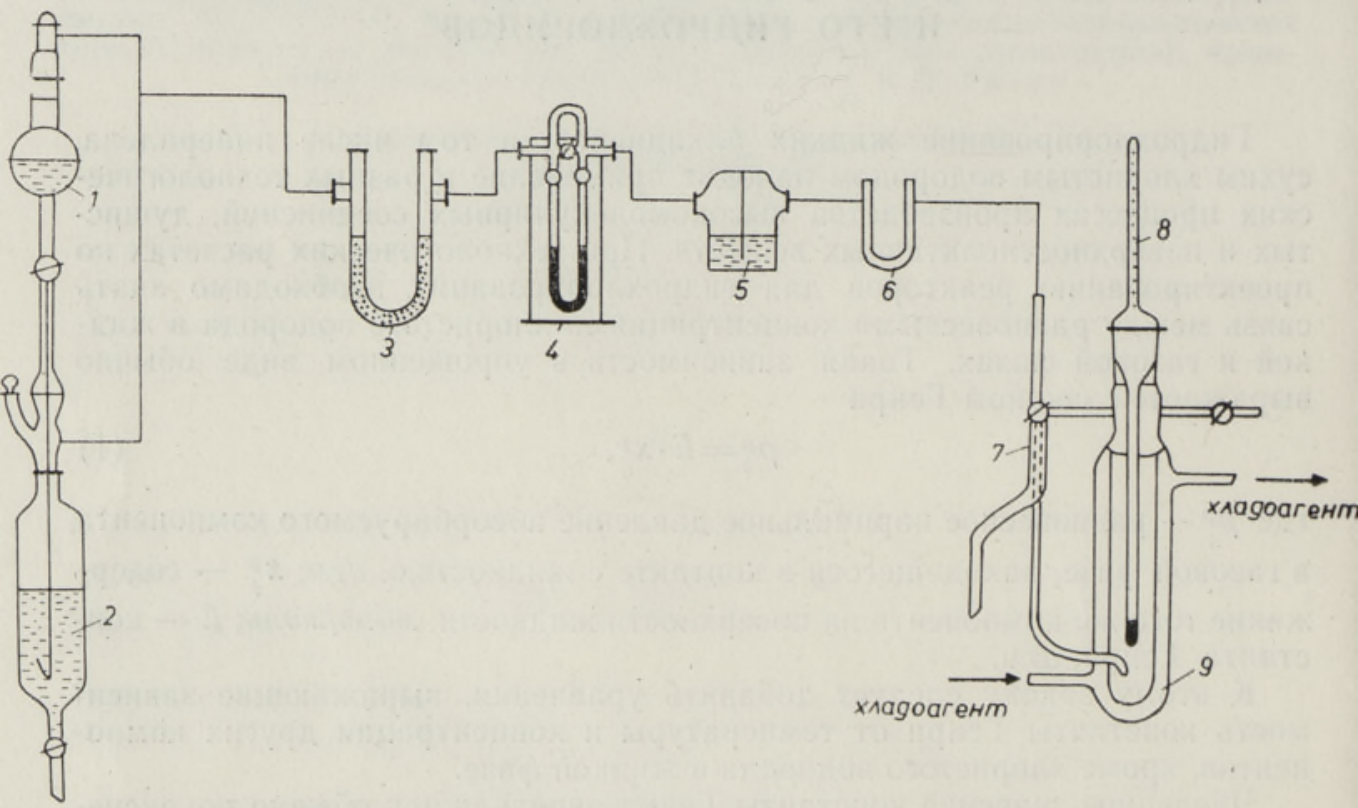

Лабораторная установка для изучения растворимости. 1 - сосуд для соляной кислоты, 2 - реактор для приготовления хлористого водорода, $3-\mathrm{CaCl}_{2}$-трубка, 4 - реометр, 5 - сосуд Тищенко с $\mathrm{H}_{2} \mathrm{SO}_{4}, 6$ - U-образная трубка, 7 - устройство для взятия проб, 8 - термометр, 9 - реактор для гидрохлорирования.

Концентрированная соляная кислота поступает из колбы (1) в реактор (2), заполненный концентрированной серной кислотой. Полученный газообразный хлористый водород проходит осушительную систему, состоящую из хлоркальциевой трубки (3) и сосуда Тищенко (5), заполненного серной кислотой: В реометре (4) определяется скорость газа, который затем проходит U-образную промежуточную емкость (6) и поступает в реактор (9).

Реактор (9) представляет собой стеклянный сосуд емкостью $60 \mathrm{M}$, снабженный термометром (8), трубкой для подачи хлористого водорода и рубашкой для термостатирующей жидкости. Устройство для взятия проб (7) имеет трехходовой кран, через который в устройство поступает газообразный хлористый водород для контакта с жидкостью, находящейся в реакторе, а также отбирается определенное количество смеси для анализа. Пробу всасывают в бюретку устройства для взятия проб (7) 
до метки, а затем ставят трехходовой кран в такое положение, чтобы проба свободно стекала в приемник.

В начале опыта проводилось насыщение предварительно охлажденной смеси продуванием большим количеством хлористого водорода. Затем температура в реакторе выравнивалась до требуемого уровня при помощи термостатирующей жидкости и уменьшения скорости газа, последняя, в свою очередь, доводилась до постоянной. После достижения стационарного режима отбирали пробы для определения состава жидкой смеси. Аналитическое содержание хлористого водорода определялось титрованием пробы, растворенной в безводном ацетоне, 0,05 н. раствором едкого калия в этиловом спирте. Содержание пиперилена и его гидрохлоридов в смеси определялось методом газовой хроматографии, а также по содержанию омыляемого хлора. Более точное описание методов прерывания реакции и анализа проб приведено в $\left[^{3,4}\right]$. Кроме того, в наших опытах проведены также измерения высоты слоя жидкости в реакторе, объемного потока газа и количества образующихся газовых пузырьков в секунду.

Опыты проводились при разных температурах и при разных содержаниях пиперилена и его гидрохлоридов (цис- и транс-2-хлор-3-пентена) в смеси. Все компоненты для приготовления исхадных смесей были предварительно очищены ректификацией. Содержание основного вещества в них превышало $99 \%$. Соляная и серная кислоты соответствовали требованиям марки «чда».

\section{Анализ экспериментальных данных}

Межфазная поверхность контакта при барботаже определяется объемной скоростью газа, диаметром пузырьков и скоростью их подъема. В данном случае имеем дело с потоком отдельных пузырьков больших размеров, скорость которых выражается уравнением [5]

$$
W=\left[\frac{4 \sigma^{2} g}{12 \pi \mu \varrho_{\varkappa}}\right]^{\frac{1}{5}},
$$

где $\sigma$ - коэффициент поверхностного натяжения, $g$ - ускорение силы тяжести, $\mu$ - вязкость и фж- плотность жидкости.

Диаметр пузырьков, несмотря на некоторое отклонение их вида от шарообразного, определяется условием

$$
d=\sqrt[3]{\frac{6 V \tau}{\pi m}},
$$

где $\tau-$ время измерения, $m-$ количество пузырьков в течение измерения, $V$ - поток газа.

Поверхность пузырьков газа на единицу объема жидкости рассчитывается формулой

$$
F=\frac{6 V H}{W d V_{*}},
$$

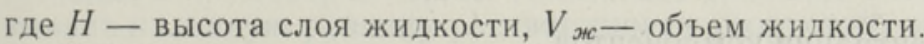

С точки зрения массопередачи в данном случае имеем дело со сложным явлением - многокомпонентной разнонаправленной диффузией как в жидкой, так и в газовой фазах. Потоки хлористого водорода и жидких 
компонентов имеют разные направления. Задача осложняется еще и тем, что скорости потоков у пиперилена и его гидрохлоридов разные из-за больших разностей давлений паров. Скорость потока пиперилена выше, чем у его гидрохлоридов. Вследствие этого, хотя общий поток гидрохлоридов и направлен в сторону жидкости, концентрация их в жидкости у поверхности иногда даже выше, чем в глубине, т. е. концентрационный градиент в жидкости направлен против общего диффузионного потока.

Точной и вполне надежной разработки общей теории диффузии для такого случая пока нет. Для решения задач, связанных с многокомпонентной диффузией в случае газов, исходят из кинетической теории газов и уравнения Максвелла. Многие исследователи считают возможным применять аналогичные уравнения и для жидкой фазы [6]. Во всяком случае, этот вариант дает основу для расчета мпогокомпонентной диффузии в жидкости, хотя и получается довольно сложная математическая модель, применение которой без ЭВМ практически невозможно. При решении задачи следует ввести некоторые допущения.

Во-первых, процесс следует считать квазистационарным, т. е. концентрации компонентов в жидкости в течение опыта не изменяются, и скорость реакции равна скорости абсорбции. Во-вторых, считаем, что вследствие медленной скорости химической реакции, в течение контакта жидкости с газом химического превращения практически не происходит. Допустимость этого условия можно легко проверить.

Таким образом, здесь нет необходимости учитывать ускорение физической абсорбции за счет химической реакции. В случае поднимающихся пузырьков коэффициент массопередачи в жидкой фазе для компонента $i$ выражается уравнением

$$
\beta_{i}^{\prime}=1,13 \sqrt{\frac{\delta_{i}^{\prime} c_{\mathrm{cp}}}{\theta}}
$$

где $\boldsymbol{\beta}_{i}^{\prime}-$ коэффициент массоотдачи компонента $i, \delta_{i}^{\prime}-$ динамический коэффициент диффузии компонента $i$ через другие, $c_{\mathrm{cp}}-$ средняя суммарная концентрация всех компонентов, $\theta=\frac{d}{w}-$ время контакта.

Массоотдача в газовой фазе определяется условием $S h=6,6$, что дает

$$
\beta_{i}^{\prime}=\frac{6,6 \delta_{i}^{\prime}}{d} \text {. }
$$

Динамические коэффициенты диффузии выражаются уравнениями

$$
\delta_{i}^{\prime}=\frac{x_{\text {пл }}}{\sum_{\substack{j=1 \\ j \neq i}}^{N} \frac{x_{j}}{\delta_{i j}^{\prime}}-\sum_{\substack{j=1 \\ j \neq i}}^{N} \frac{v_{j}}{\delta_{i j}^{\prime}}},
$$

где $v_{j}=\frac{N^{\prime}}{N_{i}^{\prime}}, N_{i}^{\prime}, N_{j}^{\prime}$ - плотности потоков диффундируемых компонентов, $x_{i}, x_{j}-$ концентрации компонентов.

Разбавление слоя выражается уравнением

$$
x_{\text {пл }}=1+\varepsilon_{i} x_{i},
$$




$$
\text { где } \varepsilon_{i}=-\sum_{i=1}^{N} v_{i} \text {. }
$$

Парные динамические коэффициенты диффузии описываются

$$
\delta_{i j}^{\prime}=D_{i j}^{\prime} c_{\mathrm{cp}}
$$

где $D_{i j}^{\prime}-$ кинематические парные коэффициенты диффузии.

Уравнения массоотдачи для отдельных компонентов выражаются в виде

$$
N_{i}^{\prime}=\beta_{i}^{\prime} \frac{\Delta x_{i}}{\left(x_{\text {пл }}\right)_{\mathrm{ep}}},
$$

где $\left(\mathrm{x}_{п л}\right)_{\text {ср }}-$ среднее логарифмическое значение разбавления слоя между рассматриваемыми сечениями.

Приведенная методика выражения диффузии и массоотдачи используется нами как для газовой, так и жидкой фазы. Плотность потока хлористого водорода определяется скоростью химического превращения в жидкости $\left[{ }^{4}\right]$. При определении плотностей потоков пиперилена и его гидрохлоридов сделано допущение, что концентрации их при выходе из аппарата равны равновесным концентрациям. Справедливость этого допущения проверена в опытах.

На основе экспериментальных данных определялись равновесные концентрации компонентов на поверхности контакта фаз. Для этого требовалось одновременное решение методом последовательного подбора системы из уравнений диффузии и массоотдачи в жидкой и газовой фазах для всех компонентов. Соответствующая итерационная процедура проводилась с помощью ЭВМ «Видеотон 1010 Б». Программа составлена на алгоритмическом языке FORTRAN.

На основе полученных данных по равновесным концентрациям хлористого водорода в жидкой и газовой фазах можно согласно уравнению (1) определить значение константы Генри в зависимости от температуры и состава жидкой смеси. Самым подходящим уравнением для выражения корреляционной связи между константой Генри, температурой и составом жидкой смеси в данном случае оказалось

$$
\ln E=A+B\left(\frac{1}{T}\right)+C x
$$

где $T$ - температура, K, $x$ - мольная доля пиперилена в жидкости.

Справедливость этого корреляционного уравнения на основе результатов 50 опытов характеризуется общим коэффициентом корреляции $R=0,96$ и частными коэффициентами $\varrho_{\ln E \frac{1}{T} \cdot x}=0,95$ и $\varrho_{\ln E x \cdot \frac{1}{T}}=0,69$. Нужно отметить практическое отсутствие корреляции между $\ln E$ и $\frac{x}{T}$, что указывает на незначительную разницу тепловых эффектов растворения хлористого водорода в пиперилене и его гидрохлоридах.

Численные значения регрессионных коэффициентов уравнения (11) $A=8,68, B=-1760, C=0,236$. Невысокое значение коэффициента $C$ указывает на малую разницу растворимости хлористого водорода в пиперилене и в его гидрохлоридах.

На основе полученных данных можно рассчитать приближенное значение теплового эффекта растворения $H=-14630$ Дж/моль. 


\section{Выводы}

1. Доказана применимость кинетической теории Максвелла для решения сложной задачи многокомпонентной противонаправленной диффузии при абсорбции хлористого водорода в реагирующей с ним жидкой системе легколетучих компонентов.

2. Рассчитаны равновесные концентрации хлористого водорода на поверхности фаз.

3. Найдено уравнение, выражающее зависимость константы Генри от температуры и состава жидкой смеси.

\section{Л И ТЕРА Т У Р А}

1. Ma nogue, W. H., Pigford, R. L., AIChE J., 6, 494 (1960).

2. Sharma, M. M., D a nckwerts, P. V., Chem. Engng Sci., 18, 729 (1963).

3 Р аяло Г., С а вич Т., Д ем ентьев а В., Аннус А., Л ээ т с К., Изв. АН ЭССР, Хим. Геол., 19, 309 (1970).

4. Р аяло Г., Д ем ентьева В., Тяхт Р., Кудрявцев И., Лээтс К., Изв. АН ЭССР, Хим. Геол., 21, 310 (1972).

5. Л е в и ч В. Г., Физико-химическая гидродинамика, М., 1960.

6. Х о б ле р Т., Массопередача и абсорбция. М., 1964.
Институт химии
Академии наук Эстонской ССР
Поступила в редакцию $30 / \mathrm{V} 1975$

Tiia SAVIC, V. DEMENTJEVA, G. RAJALO

\section{VESINIKKLORIIDI TASAKAALULINE LAHUSTUVUS \\ TEMAGA REAGEERIVATES PIPERULEENIST JA PIPERULEEN- HUDROKLORIIDIDEST KOOSNEVATES SUSTEEMIDES}

Esitatakse katsemetoodika, mille abil saab uurida vesinikkloriidi lahustuvust piperüleenis, tema hüdrokloriidides ja nende segudes. Mitmekomponendilist erisuunalist difusiooni vesinikkloriidi absorptsioonil temaga reageerivasse kergesti lenduvasse vedelikku kirjeldatakse Maxwelli kineetilise teooria abil. Katseandmete alusel on arvutatud vesinikkloriidi tasakaalukontsentratsioonid faaside piirpinnal. Nende pōhjal on leitud Henry konstandi olenevus temperatuurist ja vedela faasi koostisest. Esitatakse vastav regressioonivōrrand.

Tiia SAVICH, V. DEMENTYEVA, G. RAJALO

\section{THE EQUILIBRIUM SOLUBILITY OF HYDROGEN CHLORIDE IN SYSTEMS REACTING WITH IT} AND CONSISTING OF PIPERYLENE AND PIPERYLENE HYDROCHLORIDE

A method of experiments for studying the solubility of hydrogen chloride in piperylene, in its hydrochlorides, and in mixtures consisting of these components is being presented.

For describing the phenomena of the multicomponent countercurrent diffusion by the process of absorption of hydrogen chloride in the volatile liquid reacting with it, Maxwell's kinetical theory was used.

The equilibrium concentrations of hydrogen chloride in the interfacial area were calculated by means of digital computer. On the ground of the equilibrium concentrations obtained, the value of Henry's constant in dependence on temperature and concentrations of components of liquid phase is evaluated. The corresponding regression equation is presented. 